\title{
16 Zivil- und strafrechtliche Folgen fahrlässiger Datenschutzverstöße
}

Welche zivil- und strafrechtlichen Folgen sind im Falle eines fahrlässigen Datenschutzverstoßes vorgesehen? Geben Sie einen Überblick über die entsprechenden Regelungen des Datenschutzrechts und anderer relevanter Rechtsbereiche. Stellen Sie auch die aktuelle Rechtspraxis dar. Beispielhaft kann eine unerlaubte Re-Identifikation eines betroffenen Probanden mit ggf. sich daraus ergebender Schweigepflichtverletzung betrachtet werden.

Hinsichtlich der zivil- und strafrechtlichen Folgen von fahrlässigen Datenschutzverstößen ist in Bezug auf nicht-öffentliche Stellen aufgrund des bundeseinheitlichen Zivil- und Strafrechts keine Unterscheidung nach Bundesländern erforderlich. Soweit besondere landesrechtliche Haftungsvorschriften für öffentliche Stellen bestehen, ${ }^{952}$ werden diese in der folgenden Betrachtung ausgenommen, da diese nur graduelle Unterschiede zu den hier behandelten Vorgaben aufweisen. ${ }^{953}$

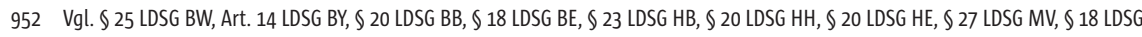

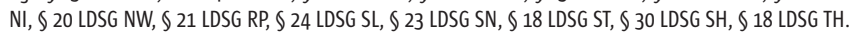

953 Vgl. insoweit die Darstellung bei Quaas, in: Wolff/Brink (Hrsg.), BeckOK BDSG, Stand 01.05.2013, Ed. 6, § 7 Rdnr. 3. 


\subsection{Zivilrechtliche Folgen}

\subsection{1 § 7 BDSG - Verschuldensabhängige Haftung}

\subsubsection{1 Überblick}

Als Grundnorm für eine zivilrechtliche Haftung für (fahrlässige) Datenschutzverstöße sieht das BDSG 57 S. I BDSG vor. Dieser gilt sowohl gegenüber öffentlichen als auch gegenüber nicht-öffentlichen Stellen und ist auf Schadenersatz und Unterlassen gerichtet. Die Vorschrift ist weder abschließend noch ausschließlich gegenüber anderen Haftungsnormen zu verstehen. ${ }^{954}$ In der Praxis kommt ihr, wie den Haftungsnormen für Datenschutzverstöße insgesamt, lediglich eine eingeschränkte Bedeutung zu. ${ }^{955}$ So existieren insbesondere keine auf Grundlage des $\mathbb{7}$ BDSC ergangenen und veröffentlichten Gerichtsentscheidungen. ${ }^{956}$

Nach $\mathbb{7}$ S. 1 BDSG ist der Träger einer verantwortlichen Stelle dem Betroffenen zum Ersatz des Schadens verpflichtet, der dem Betroffenen infolge eines unzulässigen oder unrichtigen Datenumgangs entsteht. Träger der verantwortlichen Stelle ist im nicht-öffentlichen Bereich grundsätzlich die verantwortliche Stelle selbst und in Konstellationen einer Datenverarbeitung durch öffentliche Stellen die übergeordnete Entität (Bund, Land). ${ }^{957}$ Unzulässig, d.h. rechtswidrig ist die Verarbeitung, wenn sie gegen datenschutzrechtliche Bestimmungen verstößt, worunter nicht nur solche des BDSG zu verstehen sind. So könnte beispielsweise eine Re-Identifikation eines Probanden unter dieses Merkmal fallen, wenn hierfür kein Erlaubnistatbestand besteht. Ein unrichtiger Datenumgang liegt vor, wenn Daten falsch oder unvollständig verarbeitet werden, so dass falsche Aussagen über den Betroffenen getroffen werden. 958

Anspruchsberechtigt ist ausschließlich der Betroffene als natürliche Person, der einen Schaden erlitten hat. ${ }^{959}$ Die Vorschrift ist als Verschuldenshaftung konzipiert, sodass die unzulässige oder unrichtige Datenverarbeitung vorsätzlich oder fahrlässig erfolgen muss. Unter Fahrlässigkeit ist nach $\$ 276$ Abs. 2 BGB grundsätzlich das Außerachtlassen der im Verkehr erforderlichen Sorgfalt zu sehen. Diese Vorgabe wird von $\$ 7$ BDSG insoweit modifiziert, als die verantwortliche Stelle die nach den Umständen des Falls gebotene Sorgfalt beachten muss, wodurch ein noch weitergehender Sorgfaltsmaßstab begründet wird. ${ }^{960}$

Ein Rechtsirrtum (falsche Annahme, rechtmäßig zu handeln) lässt im Zivilrecht zwar den Vorsatz für die rechtswidrige Handlung entfallen, begründet dort nach allgemeinen Grundsätzen allerdings einen Fahrlässigkeitsvorwurf, der nur dann entfällt, wenn der Irrtum unvermeidbar war. ${ }^{961}$ Dies wäre der Fall, wenn den Schädiger keine

954 Gabel, in: Taeger/Gabel (Hrsg.), BDSG, \7 Rdnr. 5; Gola/Schomerus, BDSG, § 7 Rdnr. 16.

955 Becker, in: Plath (Hrsg.), BDSG, $\$ 7$ Rdnr. 1.

956 Däubler, in: Däubler/Klebe/Wedde/Weichert (Hrsg.), BDSG, \& 7 Rdnr. 3; Quaas, in: Wolff/Brink (Hrsg.), BeckOK BDSG, Stand 01.05.2013, Ed. 6, 87 Rdnr. 3 .

957 Vgl. Gola/Schomerus, BDSG, \$ 7 Rdnr. 14

958 Ambs, in: Erbs/Kohlhaas (Hrsg.), Strafrechtliche Nebengesetze, BDSG, \$ 7 BDSG Rdnr. 3f.; Gabel, in: Taeger/Gabel (Hrsg.), BDSG, \$ 7 Rdnr. 8.

959 Vgl. Gola/Schomerus, BDSG, § 7 Rdnr. 6; Simitis, in: Simitis (Hg.), BDSG, § 7 Rdnr. 9.

960 Däubler, in: Däubler/Klebe/Wedde/Weichert (Hrsg.), BDSG, $\$ 7$ Rdnr. 15; Gabel, in: Taeger/Gabel (Hrsg.), BDSG, $\$ 7$ Rdnr. 12.

961 Vgl. Lorenz, in: Bamberger/Roth (Hrsg.), BeckOK BGB, Stand: 01.03.2011, Edition: 30, \276 Rdnr. 11ff. Anders im Strafrecht, wo ein Verbotsirrtum den (tatbestandlichen) Vorsatz unberührt lässt und sich die Frage nach der Vermeidbarkeit des Irrtums als eigener Prüfungspunkt stellt, s.u. S. $339 f$. 
Schuld daran träfe, dass er dem Irrtum unterlegen ist. Folgt er beispielweise bei zwei entgegenstehenden Rechtsmeinungen ohne weitere Beratung der einen oder lässt er sich bei Zweifeln überhaupt nicht beraten, war sein Irrtum vermeidbar. ${ }^{962}$

Von Bedeutung ist dabei, dass $\mathbb{} 7$ Abs. 2 BDSG eine Beweislastumkehr vorsieht, wonach bei einem rechtswidrigen Datenumgang vermutet wird, dass dieser schuldhaft erfolgte. Nach allgemeinen Beweislastregeln müsste der Anspruchsteller, also der Betroffene, der sich in seinen Datenschutzrechten verletzt sieht, alle Anspruchsvoraussetzungen, d.h. auch das Verschulden der verantwortlichen Stelle, beweisen, was ihm durch die angesprochene Umkehrvorschrift abgenommen wird. Allerdings kann sich die verantwortliche Stelle exkulpieren, wenn ihr ein Entlastungsbeweis gelingt, d.h. sie beweisen kann, dass sie die nach den Umständen des Falls gebotene Sorgfalt angewandt hat. Dahingegen ist die Exkulpationsmöglichkeit des $\mathbb{} 831$ BGB für das Fehlverhalten der Mitarbeiter der verantwortlichen Stelle ausgeschlossen, da $\mathbb{} 7$ die verantwortliche Stelle als geschlossene Haftungseinheit ansieht, ${ }^{963}$ ohne dass eine eigene Haftung unmittelbar gegen Mitarbeiter der verantwortlichen Stelle, den betrieblichen Datenschutzbeauftragten oder sonstige „Controller“ möglich wäre. ${ }^{964}$

Der aus der rechtswidrigen Datenverarbeitung ursächlich entstandene Schaden umfasst alle materiellen Beeinträchtigungen, worunter nur Vermögensschäden, nicht jedoch immaterielle Schäden fallen. ${ }^{965}$

\subsubsection{Auftragsdatenverarbeitung}

Diese Maßstäbe gelten auch für die Haftung bei Datenverarbeitungen im Auftrag. Die volle Verantwortung verbleibt hier bei dem Auftraggeber, der weiterhin als verantwortliche Stelle im Sinne von $\sqrt{3}$ Abs. 7 BDSG anzusehen ist und sich das Verhalten des Auftragnehmers nicht - wie üblich - nach $\$ 278$ BGB, sondern unmittelbar nach $\mathbb{} \$ 1$ BDSG zurechnen lassen muss. Ein Schadenersatzanspruch nach $\mathbb{7}$ BDSG ist demnach in Konstellationen einer Auftragsdatenverarbeitung ausschließlich gegen den Auftraggeber zu richten. ${ }^{966}$ Lediglich in Fällen, in denen sich der Auftragnehmer zum „Datenherr" aufschwingt, d.h. die Daten entgegen den Weisungen des Auftraggebers verarbeitet, kommt seine eigene Inanspruchnahme durch den Betroffenen in Betracht. ${ }^{967}$

\subsection{2 $§ 8$ BDSG - Gefährdungshaftung für öffentliche Stellen}

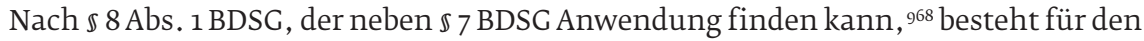
Träger öffentlicher Stellen eine verschuldensunabhängige Haftung für eine unzuläs-

962 Vgl. Unberath, in Bamberger/Roth (Hg.), BGB, § 276 Rdnr. 30, § 286 Rdnr. $56 f f$.

963 Däubler, in: Däubler/Klebe/Wedde/Weichert (Hrsg.), BDSG, Kommentar, \$ 7 Rdnr. 15; Gabel, in: Taeger/Gabel (Hrsg.), BDSG, $\$ 7$ Rdnr. 13; a.A. Becker, in: Plath (Hrsg.), BDSG, $\$ 7$ Rdnr. 17.

964 Däubler, in: Däubler/Klebe/Wedde/Weichert (Hrsg.), BDSG, \& 7 Rdnr. 8f.; Quaas, in: Wolff/Brink (Hrsg.), BeckOK BDSG, Stand 01.05.2013, Ed. 6, § 7 Rdnr. 43

965 Gabel, in: Taeger/Gabel (Hrsg.), BDSG, \$ 7 Rdnr. 10; Gola/Schomerus, BDSG, \$ 7 Rdnr. 12.

966 Vgl. Gabel, in: Taeger/Gabel (Hrsg.), BDSG, $\$ 7$ Rdnr. 15; Spoerr, in: Wolff/Brink (Hrsg.), BeckOK BDSG, Stand: 01.11.2013, Ed. 6, \11 Rdnr. 83.

967 Simitis, in: Simitis (Hg.), BDSG, \ 7 Rdnr. 11; Quaas, in: Wolff/Brink (Hg.), BeckOK BDSG, Stand 01.05.2013, Ed. 6, \7 Rdnr. 41.

968 Gabel, in: Taeger/Gabel (Hrsg.), BDSG, §8 Rdnr. 4. 
sige oder unrichtige automatisierte Verarbeitung von personenbezogenen Daten. Da es sich bei $₫ 8$ BDSC um eine Gefährdungshaftung handelt, die kein Verschulden voraussetzt, und die Tatbestandsvoraussetzungen damit umso leichter zu bejahen sind, sieht $\mathbb{8}$ Abs. 3 BDSG eine Begrenzung der Haftungssumme auf 130.000 Euro vor. Bei einer schweren Verletzung des Persönlichkeitsrechts hat der Betroffene nach $\mathbb{s} 8$ Abs. 3 BDSC im Gegensatz zu $\mathbb{7}$ BDSG einen Anspruch auf ein angemessenes Schmerzensgeld. In Fällen, in denen mehrere öffentliche Stellen gemeinsam Zugriff auf einen Datenpool haben, kann jede dieser Stellen der Haftung unterliegen, wenn sich die speichernde Stelle nicht feststellen lässt ( 8 Abs. 4 BDSG).

\subsection{3 $\ 823$ Abs. 1 und 2 BGB - Verschuldensabhängige Haftung}

Da das allgemeine Persönlichkeitsrecht des Betroffenen als „sonstiges Recht“ grundsätzlich gegen jegliche deliktische Beeinträchtigungen geschützt ist, können sich Schadenersatzansprüche bei schuldhafter Verletzung auch aus $\$ 823$ Abs. 1 BGB ergeben. Dieser umfasst auch immaterielle Schäden, ${ }^{969}$ greift in der Praxis der Gerichte aufgrund geringer Fallzahlen aber nur sehr selten durch. ${ }^{970}$ Dabei sind Ansprüche

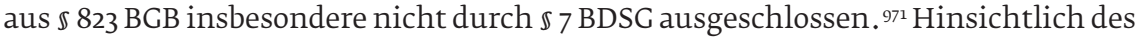

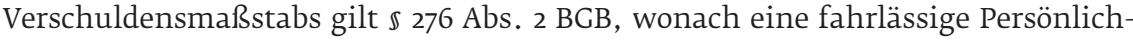
keitsverletzung in Betracht kommt, wenn der Handelnde die im Verkehr erforderliche Sorgfalt außer Acht lässt, ihn also z.B. auch ein Organisationsverschulden trifft.

Wird eine datenschutzrechtliche Vorschrift verletzt, die nicht nur allgemeinen Interessen, sondern vielmehr gerade dem individuellen Schutz des Betroffenen zu dienen bestimmt ist, also als Schutzgesetz im Sinne von $\$ 823$ Abs. 2 BGB anzusehen ist, so käme auch hieraus ein Anspruch in Betracht. Im Einzelnen ist allerdings umstritten, welchen Normen der Charakter eines Schutzgesetzes zukommen kann. Vertreten wird insbesondere, dass jede datenschutzrechtliche Norm ein Schutzgesetz darstellen soll. 972

Der Anspruch kann auch gegen eine juristische Person geltend gemacht werden. Dann müsste sich diese das schuldhafte Handeln ihrer Organe nach den $\$ \$ 30,31,89$ BGB zurechnen lassen können.

\subsection{4 $§ 280$ Abs. 1 in Verbindung mit $§ 241$ Abs. 2 BGB - Vertragliche Haftung}

Daneben kommt auch eine vertragliche Haftung auf Schadensersatz in Betracht, ${ }^{973}$ denn eine rechtswidrige Datenverarbeitung bedeutet regelmäßig zugleich auch die Verletzung einer vertraglichen Nebenpflicht des (Behandlungs-)Vertrags. ${ }^{974}$ Ein Verschulden der verantwortlichen Stelle würde hier - ähnlich wie bei $\mathbb{} 7$ Abs. 2 BDSG -

969 Gabel, in: Taeger/Gabel (Hrsg.), BDSG, § 7 Rdnr. 10.

970 Vgl. die Darstellung bei Gabel, in: Taeger/Gabel (Hrsg.), BDSG, \& 7 Rdnr. 26.

971 Gola/Schomerus, BDSG, \ 7 Rdnr. 18a.

972 Vgl. Däubler, in: Däubler/Klebe/Wedde/Weichert (Hrsg.), BDSG, \& 7 Rdnr. 33; Gola/Schomerus, BDSG, § 7 Rdnr. 18b; differenzierend Gabel, in: Taeger/Gabel (Hrsg.), BDSG, \& 7 Rdnr. 27.

973 Insoweit auch zugunsten juristischer Personen, was von Bedeutung sein kann, wenn die verantwortliche Stelle beispielsweise be einem Auftragsdatenverarbeiter wegen eines Datenlecks Rückgriff nehmen möchte.

Gola/Schomerus, BDSG, \& 7 Rdnr. 18. 
nach $₫ 28$ o Abs. 1 S. 2 BGB vermutet werden und könnte nur über einen Entlastungsbeweis abgewehrt werden. Das Verhalten von zur Erfüllung eingesetzten Personen

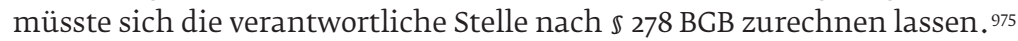

\subsubsection{Sonstige Haftungsnormen}

Im Übrigen besteht parallel zu den obigen Vorschriften mit den Bestimmungen des Staatshaftungsrechts ( $\$ 839$ BCB, Art. $34 \mathrm{CG}$ ) ein weiteres Haftungsregime für öffentliche Stellen bzw. deren Bedienstete, für die $\$ 823$ BCB nicht gilt. ${ }^{776}$ Für nicht öffentliche Stellen ist ferner auf $₫ 831$ BCB zu verweisen, wonach eine verantwortliche Stelle auch für das Fehlverhalten ihrer Mitarbeiter haften kann. Prinzipiell kann schließ-

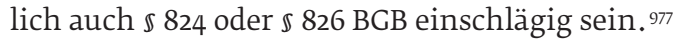

\subsection{Strafrechtliche Folgen}

Strafrechtliche Sanktionen für Datenschutzverstöße sehen insbesondere $\mathbb{4} 44$ BDSC und $\$ 203$ StCB vor. Die Vorschriften setzen aber zumindest eine (bedingt) vorsätzliche Begehungsweise voraus ( $\$ 15$ StCB), so dass fahrlässige Verstöße gegen datenschutzrechtliche Vorgaben strafrechtlich nicht sanktioniert werden. Dass nur fahrlässig gegen Datenschutzvorschriften verstoßen wird, strafrechtlich aber (bedingter) Vorsatz etwa in Bezug auf eine Verletzung der Verschwiegenheitspflicht aus $\mathbb{2} 203$ StGB anzunehmen wäre, erscheint eher fernliegend.

\subsubsection{Abgrenzung: Bedingter Vorsatz und bewusste Fahrlässigkeit}

Unter bedingtem Vorsatz im strafrechtlichen Sinne ist zu verstehen, dass der Täter handelt, obwohl er die hinreichend wahrscheinliche Möglichkeit des Eintritts eines Straftatbestandes ernstlich erkennt, diesen aber billigend in Kauf nimmt. ${ }^{778}$ Hiervon abzugrenzen ist die bloße bewusste Fahrlässigkeit, bei der der Täter die Möglichkeit gleichsam erkennt, auf den Nichteintritt des strafbaren „Erfolges“ aber sicher vertraut.

\subsubsection{Abgrenzung: Normativer Verbotsirrtum und faktische Fahrlässigkeit}

$\mathrm{Zu}$ beachten ist allerdings, dass ein Irrtum bzw. die Nachlässigkeit auf normativer Ebene (unrichtige Annahme, rechtmäßig zu handeln) im Strafrecht nicht zu bloßer Fahrlässigkeit führt, sondern den Vorsatz für eine bestimmte Datenverarbeitung unberührt lässt. ${ }^{979}$ Hier würde sich allerdings die Frage stellen, welche sonstigen, eine Sanktion ausschließenden oder abmildernden Folgen der dann vorliegende Verbotsirrtum hat.

975 Gabel, in: Taeger/Gabel (Hrsg.), BDSG, $\$ 7$ Rdnr. 24.

976 Gola/Schomerus, BDSG, $\$ 7$ Rdnr. 17.

977 Näher dazu Simitis, in: ders. (Hrsg.), BDSG, $\$ 7$ Rdnr. 68.

978 Hierzu und zur Abgrenzung von der bewussten Fahrlässigkeit: Fischer, StGB, $\ 15$ Rdnr. gff.

979 Anders als im Zivilrecht, wo der Verbotsirrtum den Vorsatz ausschließt, aber zu einer möglichen Fahrlässigkeitshaftung führt, s.o. S. 336 und Vogel, in: Laufhütte/Rissing-van Saan/ Tiedemann (Hg.), Leipziger Kommentar zum StGB, \ 17, Rdnr. 120. 
Im Bereich des Strafrechts schließt ein unvermeidbarer Verbotsirrtum Schuld und damit auch Strafe aus: „Fehlt dem Täter bei Begehung der Tat die Einsicht, Unrecht zu tun, so handelt er ohne Schuld, wenn er diesen Irrtum nicht vermeiden konnte“ ( $\$ 17$ S. 1 StCB). Hätte der Täter hingegen den Irrtum vermeiden können, insbesondere durch rechtliche Beratung, kann die Strafe lediglich gemildert werden ( $\$ 17$ S. 1, $\mathbb{\$} 49$ Abs. 1 StCB), sie muss es aber nicht - dies obliegt letztlich der Würdigung des zuständigen Gerichts.

Die Anforderungen, die an den Täter bei der Prüfung der Vermeidbarkeit gestellt werden, sind höher als bei der Beurteilung der Fahrlässigkeit. Der Täter muss „alle seine Erkenntniskräfte und sittlichen Wertvorstellungen“ anstrengen. ${ }^{980}$ Ein solcher vermeidbarer Verbotsirrtum besteht beispielsweise, wenn ein Arzt ohne genaue Prüfung eine nicht gegebene Offenbarungsbefugnis annimmt. Hier ist ähnlich wie im Zivilrecht eine Vermeidbarkeit des Irrtums anzunehmen, wenn beispielweise bei zwei entgegenstehenden Rechtsmeinungen ohne weitere Beratung der einen gefolgt wird oder wenn der Täter sich bei Zweifeln überhaupt nicht beraten lässt. ${ }^{981}$

Hingegen ist der rein faktisch fahrlässig handelnde Täter straflos, da in $\mathbb{2 0 3}$ StCB lediglich die vorsätzliche Begehung unter Strafe gestellt ist. Der „Arzt, der fahrlässig seine Kartei verliert“, wird folglich nicht bestraft. ${ }^{982}$ Ein vorsätzliches Unterlassen gebotener Sicherheitsvorkehrungen, die zum Verlust von Patientendaten führen, kann jedoch strafbar sein. ${ }^{983}$

\subsection{Ergebnis}

Bei fahrlässigen Datenschutzverstößen kann die verantwortliche Stelle bzw. deren Träger zivilrechtlichen Schadenersatzansprüchen ausgesetzt sein. Eine Verschuldenshaftung besteht insoweit für öffentliche und nicht-öffentliche Stellen nach $₫ 7$ BDSG. Für öffentliche Stellen kommt sogar eine Gefährdungshaftung aus $\mathbb{8} 8$ BDSG in Betracht. Darüber hinaus gelten die allgemeinen vertragsrechtlichen $(\$ 280 \mathrm{Abs} .1$ in Verbindung mit $\mathbb{2} 241$ Abs. 2 BCB) und deliktischen ( $\$ 823$ Abs. 1, 2 BGB) Haftungsregime. Gegenüber öffentlichen Stellen können ferner die staatshaftungsrechtlichen Vorschriften greifen.

Die Bedeutung dieser Vorschriften ist in der Praxis aber eher gering, was daran liegen kann, dass Datenschutzverstöße für den einzelnen Betroffenen oft nicht feststellbar sind, i.d.R. keine bezifferbaren Schäden verursachen und diesen oft bereits durch die Betroffenenrechte (Berichtigung, Löschung etc.) wirksam begegnet werden

980 BGH, Urt. v. 23.12.1952 - 2 StR 612/50, BGHSt 4, 1, 5.

981 Vgl. oben S. 336

982 Ulsenheimer, in Laufs/Kern (Hg.), Handbuch des Arztrechts, \$ 145 Rdnr. 4.

983 Fischer, StGB, \& 203 Rdnr. 30b: „Wer aus Bequemlichkeit darauf verzichtet, seinen Schreibtisch aufzuräumen oder seinen PC vor Zugriffen zu schützen und die Kenntniserlangung Dritter in Kauf nimmt, offenbart durch Unterlassen“. Auf den ersten Blick etwas widersprüchlich insoweit Ulsenheimer, in: Laufs/Kern (Hg.), Handbuch des Arztrechts, der in $\$ 145$ Rdnr. 4 ausführt, dass ein Arzt, der seine Kartei fahrlässig nicht gegen Diebstahl sichert, nicht strafbar ist, während in $\$ 66$ Rdnr. 9 angeführt wird, das ein Offenbaren auch durch Unterlassen begangen werden kann, z.B. wenn der Arzt Patientenunterlagen unverschlossen liegen lässt. Maßgeblich wird insoweit die Abgrenzung zwischen (bewusster) Fahrlässigkeit und (bedingtem) Vorsatz sein. S. auch oben S. $48 \mathrm{f}$. 
kann. ${ }^{984}$ Eine Verurteilung auf Schmerzensgeld ( $\$ 254$ Abs. 2 BGB) ist allerdings möglich.

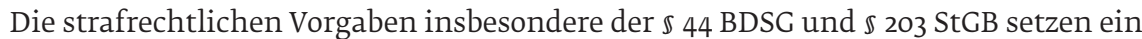
(bedingt) vorsätzliches Verhalten voraus und finden bei bloß fahrlässigen Datenschutzverstößen folglich keine Anwendung. 